\title{
The liposoluble proteome of Mycoplasma agalactiae: an insight into the minimal protein complement of a bacterial membrane
}

\author{
Carla Cacciotto ${ }^{1 \dagger}$, Maria Filippa Addis ${ }^{1,2 \dagger}$, Daniela Pagnozzi ${ }^{2}$, Bernardo Chessa' ${ }^{1}$ Elisabetta Coradduzza', \\ Laura Carcangiu', Sergio Uzzau ${ }^{2,3}$, Alberto Alberti $^{1 *+}$, Marco Pittau $^{1+}$
}

\begin{abstract}
Background: Mycoplasmas are the simplest bacteria capable of autonomous replication. Their evolution proceeded from gram-positive bacteria, with the loss of many biosynthetic pathways and of the cell wall. In this work, the liposoluble protein complement of Mycoplasma agalactiae, a minimal bacterial pathogen causing mastitis, polyarthritis, keratoconjunctivitis, and abortion in small ruminants, was subjected to systematic characterization in order to gain insights into its membrane proteome composition.

Results: The selective enrichment for $M$. agalactiae PG2 ${ }^{\top}$ liposoluble proteins was accomplished by means of Triton X-114 fractionation. Liposoluble proteins were subjected to 2-D PAGE-MS, leading to the identification of 40 unique proteins and to the generation of a reference 2D map of the M. agalactiae liposoluble proteome. Liposoluble proteins from the type strain PG2 and two field isolates were then compared by means of 2D DIGE, revealing reproducible differences in protein expression among isolates. An in-depth analysis was then performed by GeLCMS/MS in order to achieve a higher coverage of the liposoluble proteome. Using this approach, a total of 194 unique proteins were identified, corresponding to $26 \%$ of all $M$. agalactiae $P G 2^{\top}$ genes. A gene ontology analysis and classification for localization and function was also carried out on all protein identifications. Interestingly, the $11.5 \%$ of expressed membrane proteins derived from putative horizontal gene transfer events.
\end{abstract}

Conclusions: This study led to the in-depth systematic characterization of the M. agalactiae liposoluble protein component, providing useful insights into its membrane organization.

\section{Background}

Mycoplasmas are the smallest and simplest prokaryotes capable of self-replication, being provided only with the minimal machinery required for survival. During evolution, they have regressively evolved from gram-positive bacteria by reduction of their genome to an essential minimum, economizing their structural elements, metabolic pathways, and genetic resources [1].

Among other consequences, this cost-cutting strategy led to loss of the cell-wall component, and therefore to lack of a peptidoglycan "shell". Instead, sterols are incorporated into the lipid bilayer, providing resistance to

\footnotetext{
* Correspondence: alberti@uniss.it

+ Contributed equally

'Dipartimento di Patologia e Clinica Veterinaria, Università degli Studi di

Sassari, Sassari, Italy

Full list of author information is available at the end of the article
}

rupture, but still allowing a certain flexibility of cell shape. Integral and associated membrane proteins are therefore directly exposed and act as the immediate bacterial interface, playing a major role in survival and pathogenesis [2,3]. Gathering information on membrane proteins of such a pathogen might provide novel and interesting insights on its biology, and generate useful information for improving diagnosis, vaccination, and therapy. Recently, a large-scale study was carried out on the proteome of the human pathogen Mycoplasma penetrans, based on the TAP-MS approach [4]. However, membrane proteins were not included in this study, since they require dedicated protocols for purification and analysis and present numerous challenges.

Many members of the genus Mycoplasma are pathogenic for humans, animals, plants, and insects. M. agalactiae is the etiological agent of Contagious Agalactia (CA), 
a serious disease of sheep and goats characterized by mastitis, polyarthritis, keratoconjunctivitis, and abortion $[1,5,6]$. CA has a worldwide distribution and is endemic in Mediterranean Countries [7], causing severe economic losses in areas where economy is largely based on small ruminant milk production [5].

In Europe, the disease has been tentatively controlled either by vaccination or with serological tools based on recombinant surface proteins [8-13]. At present, the two above mentioned strategies are not actually compatible until proper DIVA (Differentiating Infected from Vaccinated Animals) vaccines will allow discrimination of vaccinated animals from naturally infected ones. The highly immunogenic, surface-associated membrane proteins represent key antigens for diagnosis and vaccine development. However, the finding of constantly expressed surface proteins in mycoplasmas is complicated by the existence of mechanisms aimed to evade the host immune response [1,14-17]. Surface-associated proteins are also pivotal for pathogenesis by acting as cytoadhesins [18]. To date, a limited number of constantly expressed surface proteins have been described in M. agalactiae. Among them, P30, P48, and P80 were described as antigens [19-21]; other proteins belong to the variable surface membrane proteins family (Vpma) [14,17], and P40 was suggested to play an important role in attachment to the host cell [18].

Genetic approaches traditionally used for large scale investigation of protein sets have been poorly applied to mycoplasmas. The expression of immunogenic Mycoplasma proteins in Escherichia coli expression libraries is hampered by the very high $\mathrm{A}+\mathrm{T}$ content (almost $80 \%$ ) and by the Mycoplasma-specific codon usage, resulting in abnormal internal transcription/translation and in premature termination, respectively [22,23]. In 2007, the full genome sequence of the $M$. agalactiae type strain PG2 $\left(\mathrm{PG}^{\mathrm{T}}\right)$ was published [24] and paved the way for systematic proteomic studies in mycoplasmas.

The combination of 2-D PAGE and mass spectrometry (MS) is a well-established method for the systematic and comparative study of proteomes, since it allows the simultaneous visualization and identification of the protein complement of a cell. However, it is commonly reported that standard 2-D PAGE lacks in resolution of very hydrophobic and basic proteins, which are particularly abundant in the Mycoplasma membrane [25-27]. Indeed, membrane proteins are poorly detected in 2-D PAGE maps of Mycoplasma total protein extracts $[22,28]$. Triton X-114 fractionation may assist in solving this problem, since it was demonstrated to enable a selective enrichment in hydrophobic proteins $[29,30]$. Triton $\mathrm{X}-114$ fractionation followed by 2-D PAGE remains the method of choice for proteomic characterization of the membrane protein subset [31], and for differential analysis of membrane protein expression among bacterial strains [32]. More specifically, the recently developed Differential In Gel Electrophoresis (DIGE) [33-35], based on labeling of protein samples with fluorescent dyes before 2-D electrophoresis, enables the accurate analysis of differences in protein abundance between samples. However, considering the above mentioned intrinsic limitations of 2-D PAGE, other gel-based proteomic approaches, such as one-dimensional PAGE and Liquid Chromatography-Tandem Mass Spectrometry (GeLCMS/MS) [36], can be combined with the 2-D PAGE/MS in order to mine deeper into a liposoluble proteome.

In this study, the membrane proteome of $M$. agalactiae was characterized by means of Triton X-114 fractionation, 2-D PAGE-MS, GeLC-MS/MS, and Gene Ontology classification. Differential expression of membrane proteins among $M$. agalactiae strains was also evaluated by 2 D DIGE.

\section{Results}

\section{Extraction of bacterial proteins and isolation of liposoluble proteins}

This study was aimed to the systematic characterization of $M$. agalactiae $\mathrm{PG}^{\mathrm{T}}$ membrane proteins by means of a gel-based proteomic approach. In order to increase coverage for liposoluble proteins, a commercial fractionation method and the classical Triton X-114 fractionation protocol were applied to $M$. agalactiae $\mathrm{PG}^{\mathrm{T}}$ cell lysates. The best results were obtained by means of Triton X-114 fractionation. Figure 1A illustrates the hydrosoluble and liposoluble fractions obtained from M. agalactiae $\mathrm{PG} 2^{\mathrm{T}}$, flanked by the total protein pattern for comparison. The efficiency of the procedure in separating liposoluble proteins was evaluated by Western immunoblotting using a rabbit hyperimmune serum raised against $M$. agalactiae $\mathrm{P} 48$, a previously characterized surface lipoprotein $[12,19]$. As expected, presence of $\mathrm{P} 48$ was observed only in the total extract and in the Triton X-114 phase (Figure 1B), confirming that the fractionation method enabled separation and enrichment of hydrophobic proteins.

\section{2-D PAGE/MS of $M$. agalactiae PG2 ${ }^{\top}$ liposoluble proteins}

Total proteins and the Triton X-114 soluble fraction of $M$. agalactiae $\mathrm{PG} 2^{\mathrm{T}}$ were subjected to 2-D PAGE separation in order to evaluate the extent of enrichment in basic and liposoluble proteins. As illustrated in Figure 2, left panel, a very high number of spots were present in the total protein map of $M$. agalactiae $\mathrm{PG} 2^{\mathrm{T}}$ but, as expected, basic proteins were poorly represented. Upon comparison, the 2-D PAGE map generated with the Triton X-114 soluble fraction showed a significant enrichment in basic proteins, 


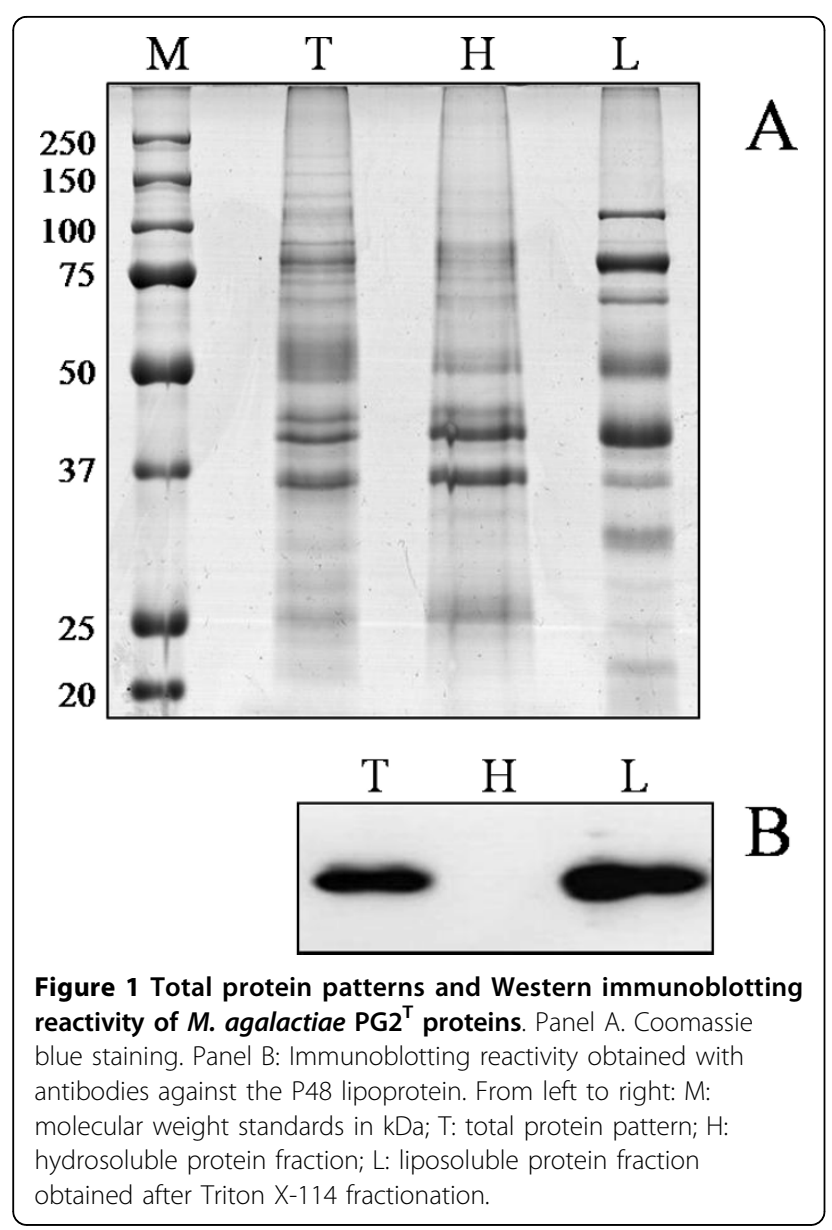

with an excellent resolution also in high-abundance spots (Figure 2, right panel).

In order to attain a systematic characterization of the liposoluble proteome, the Triton X-114 phase fraction of $M$. agalactiae $\mathrm{PG}^{\mathrm{T}}$ was subjected to 2-D PAGE under three different $p I$ intervals: 3-10NL, 7-11, and 4-7 (Additional files 1, 2, and 3). From these 2D maps, about 300 spots were excised and identified by MALDITOF and nanoHPLC-nanoESI-Q-TOF MS. This approach led to the successful identification of 40 unique proteins, corresponding to $5.4 \%$ of all $M$. agalactiae $\mathrm{PG} 2^{\mathrm{T}}$ genes. Figure 3 reports a representative liposoluble protein map summarizing the main protein identifications accomplished on 2-D spots. A detailed description of all protein identifications is given in Additional file 4. Experimentally deduced molecular weight and $p I$ of protein spots were compared with the theoretical parameters obtained from MASCOT, and most experimental data were in accordance with theoretical data. Few proteins, such as $\mathrm{VpmA}$, were detected in multiple spots at different $p I s$ and molecular weights, as expected for this class of lipoproteins which undergo size variation. The well-known immunogenic proteins $[12,17,19-21]$ were all detected by 2-D PAGE at the expected $p I$ and MW. All six variable surface lipoproteins encoded in the $M$. agalactiae $\mathrm{PG} 2^{\mathrm{T}}$ genome were also detected, some of which (such as VpmaY and VpmaD) with high expression levels, as could be expected considering their relevance in providing variability to the mycoplasmal antigenic mosaic.

\section{D DIGE of liposoluble proteins among the type strain and two field isolates of $M$. agalactiae}

In order to assess the suitability of 2-D PAGE for comparison of the membrane protein composition, the liposoluble protein profiles of $M$. agalactiae $\mathrm{PG}^{\mathrm{T}}$ and two field isolates were compared by 2D DIGE (Figure 4).

The images generated upon acquisition of the single color channels enable to evaluate the liposoluble protein

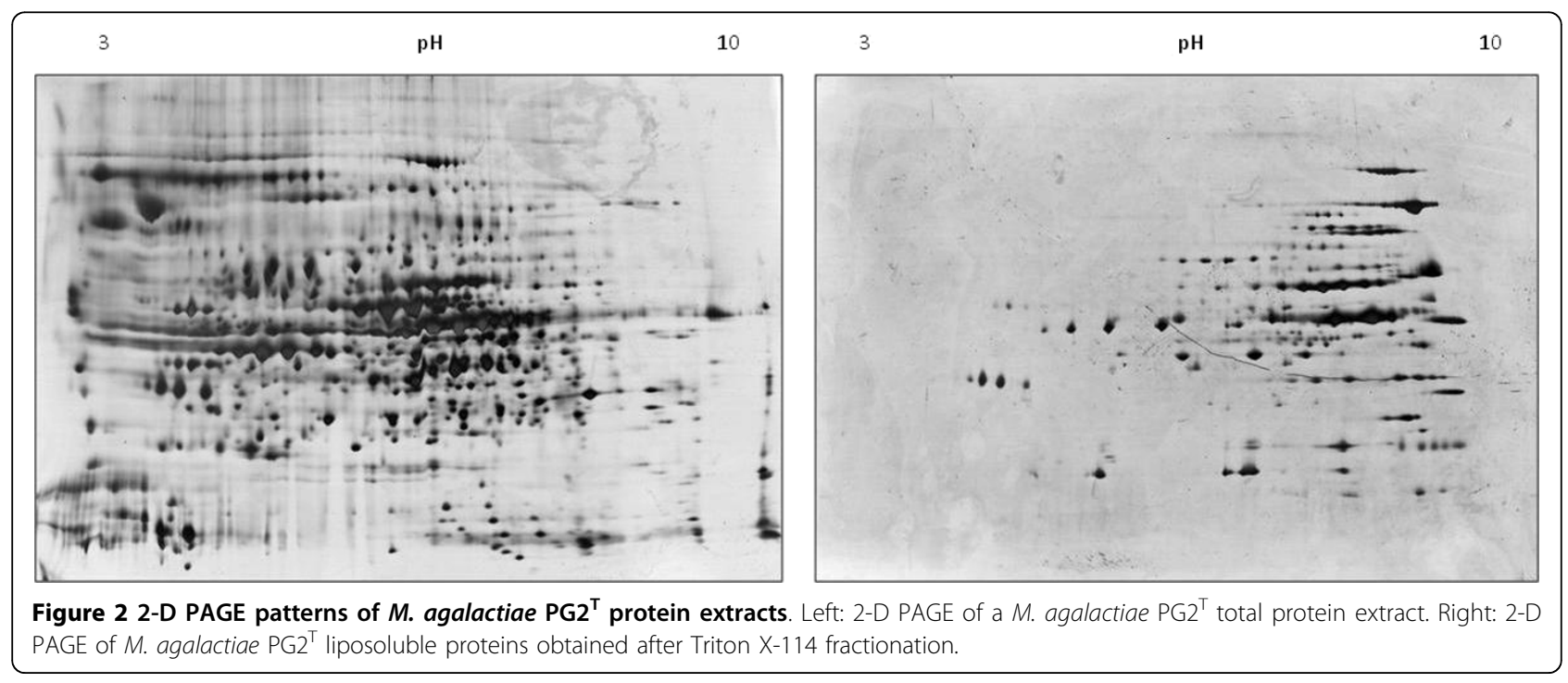




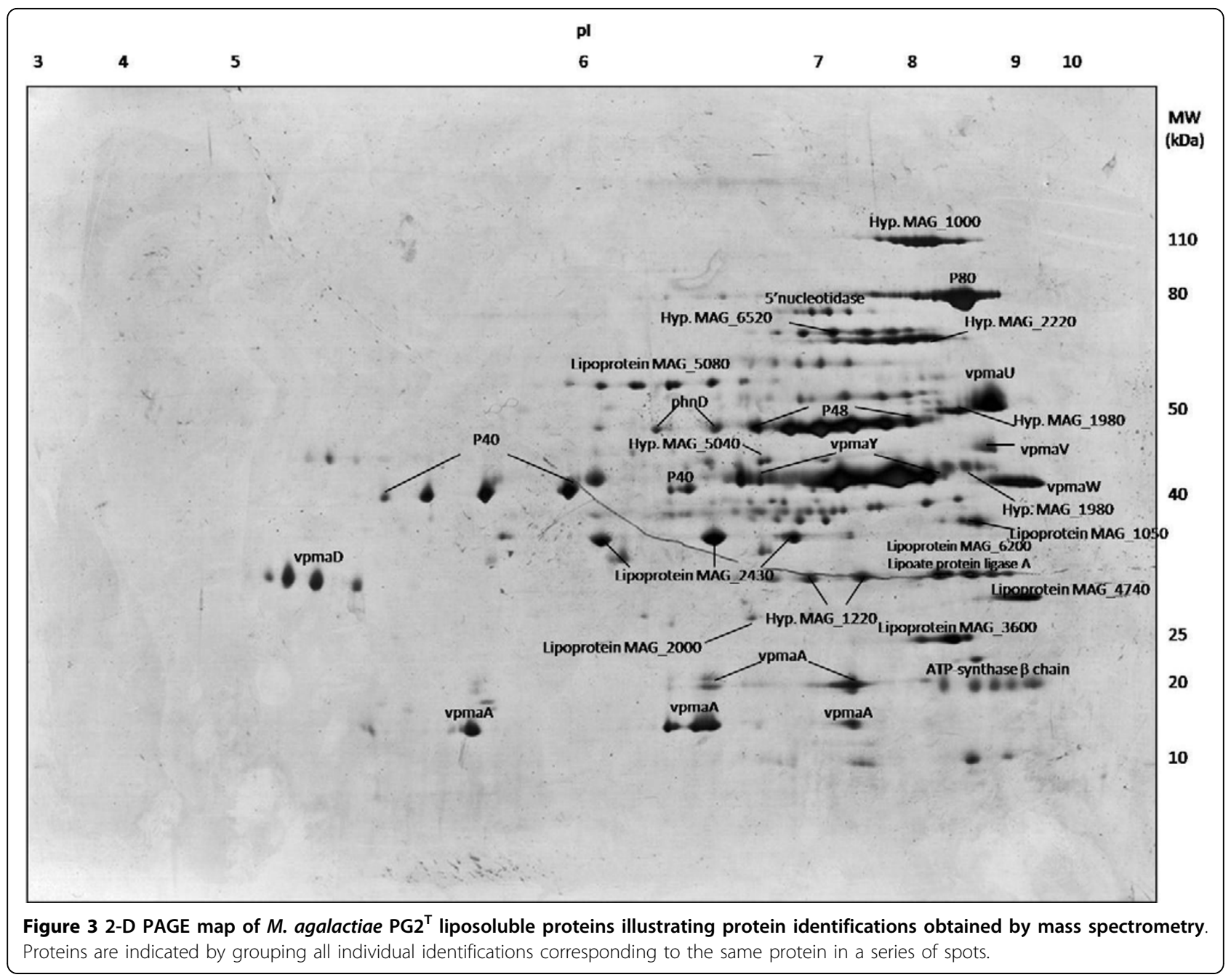

profiles separately (Figure 4, A, B, C), while comparison of two protein profiles can be performed upon superimposition of two color signals (Figure 4, D, E, F). In the overlay image, the three proteome $2 \mathrm{D}$ maps can be compared. Although many spots are shared among the three profiles (in white), a number of differences in expression can be appreciated. In fact, several spots are present only in one (blue, green, red) or two profiles (purple, yellow, light blue). Many already known antigens (such as P80, P48, P40, and most Vpmas) appear in white, indicating superimposition of the three signals and therefore presence in all three bacterial proteomes. Several differences among the three profiles can be easily observed; for example, the series of spots at 40 $\mathrm{kDa}$ corresponding to $\mathrm{VpmaY}$ (in purple in the overlay image, Figure 4) is present only in two cases $\left(\mathrm{PG}^{\mathrm{T}}\right.$ and Bortigali) while the series of spots at $23 \mathrm{kDa}$ (in green) is present only in one case (Nurri). The application of this method to an adequate number of isolates might enable to easily detect constantly expressed proteins that might serve as candidate antigens for development of vaccines and diagnostic tools.

\section{GeLC-MS/MS of $M$. agalactiae PG2 liposoluble proteins}

Although well suited for lipoprotein analysis, the 2-D PAGE/MS strategy presents drawbacks in analysis of transmembrane proteins, such as permeases or other highly hydrophobic proteins. Moreover, these protein classes may undergo selective loss during precipitation/ resolubilization steps. In order to increase the membrane protein coverage and minimize selective protein loss, SDS-PAGE and GeLC-MS/MS analysis were performed on the non-precipitated Triton X-114 liposoluble protein fraction. A total of 36 slices were cut from the SDS-PAGE gel lane containing the separated liposoluble proteins (Additional file 5) and subjected to nanoHPLC-nanoESI-Q-TOF-MS/MS identification. Upon application of this method, 194 mycoplasma proteins were identified in total, corresponding to $26 \%$ of all M. agalactiae $\mathrm{PG}^{\mathrm{T}}$ genes, 38 of which were also 


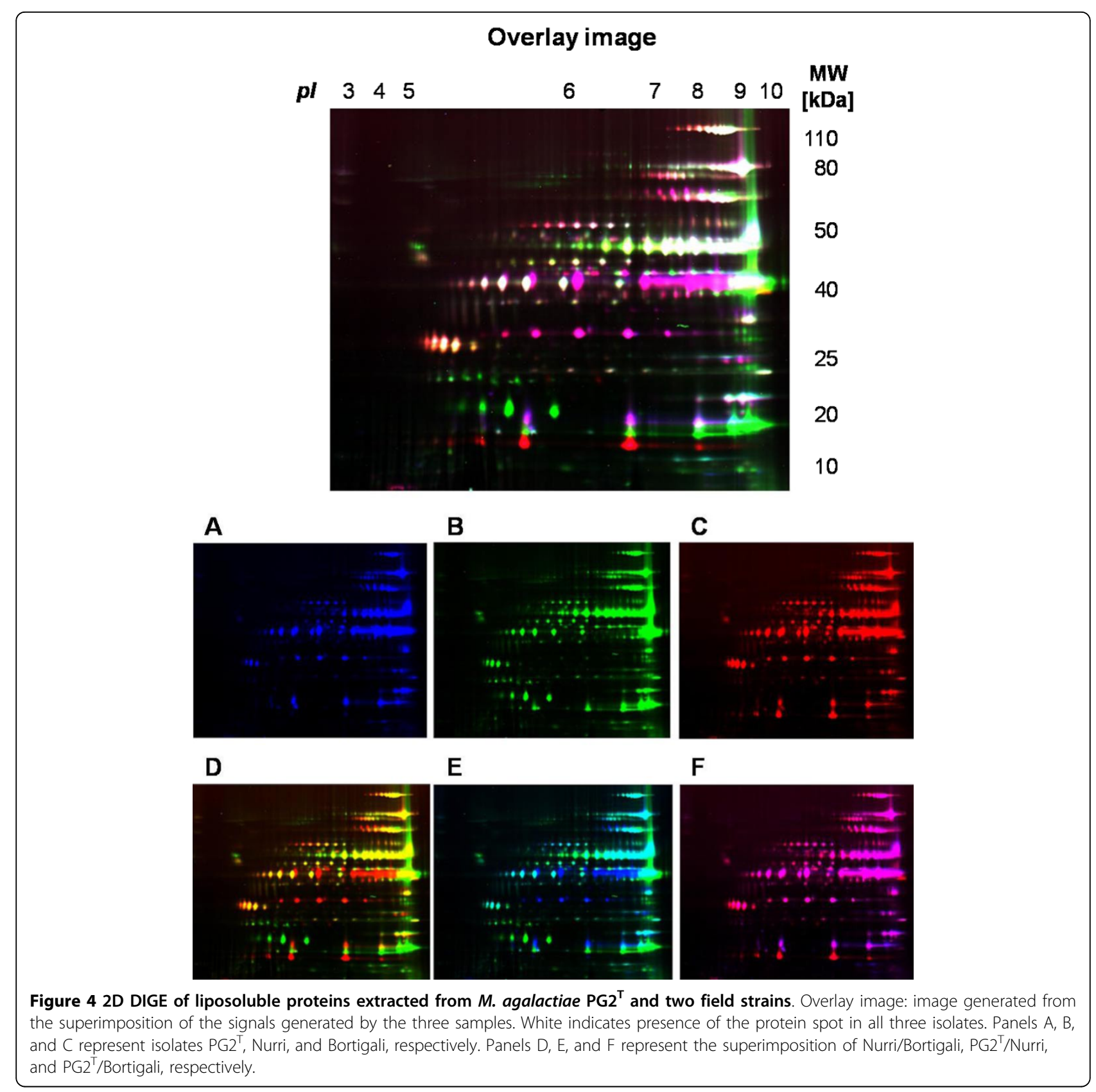

identified by 2-D PAGE/MS (for a detailed list of protein identifications, see Additional file 6; Additional file 7 reports a summary table listing all unique protein identifications).

\section{Data analysis and classification}

A gene ontology (GO) classification was carried out on proteins identified by 2-D PAGE/MS and GeLC-MS/ MS. For the first method, proteins $(n=40)$ were mostly classified by the GO software as hypothetical lipoproteins $(65 \%)$, cytoplasmic proteins $(22 \%)$, ribosomal proteins $(8 \%)$, and other membrane-located proteins (5\%).
When identifications obtained by GeLC-MS/MS were also included in the GO analysis $(\mathrm{n}=194), 43 \%$ of all identifications were assigned to proteins located on the membrane, either lipoproteins (17\%) or other membrane proteins $(26 \%)$, whereas $36 \%$ were classified as cytoplasmic, $17 \%$ as ribosomal, and $4 \%$ of unknown localization (Figure 5).

All protein identifications were then classified according to function (Figure 6, and Additional file 7). As expected, a high proportion of the identified proteins perform membrane transport functions (about 16\%), and belong mostly to $\mathrm{ABC}$ transporters (13\%). Transmembrane proteins, 


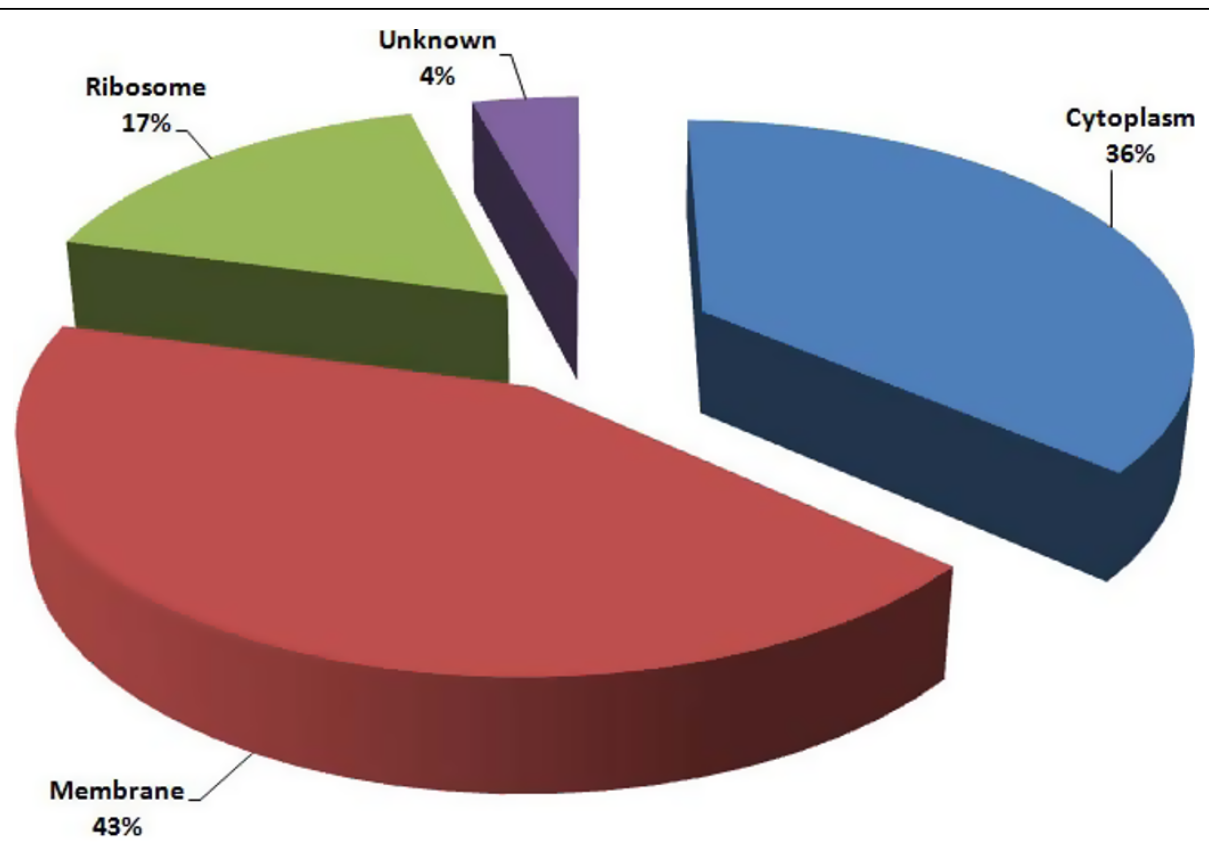

Figure $5 \mathrm{GO}$ graph of proteins identified by 2-D PAGE-MS and GeLC-MS/MS in the Triton X-114 fraction of M. agalactiae PG2 $^{\top}$. Protein identifications are classified according to cellular localization.

such as permeases, were detected only by means of GeLCMS/MS. Another highly represented functional process was translation (19\%), due to the elevated number of ribosomal proteins identified. Hydrolytic enzymes were also significantly represented (6\%), highlighting their crucial role for survival of mycoplasmas. Several other functional classes, such as enzymes involved in amino acid, carbohydrate, lipid, and nucleic acid metabolism, were significantly represented in the $M$. agalactiae $\mathrm{PG}^{\mathrm{T}}$ liposoluble protein fraction. Secretion/export systems accounted for $4 \%$ of all identified proteins; these components are in fact crucial for maturation and release of secreted proteins, but also

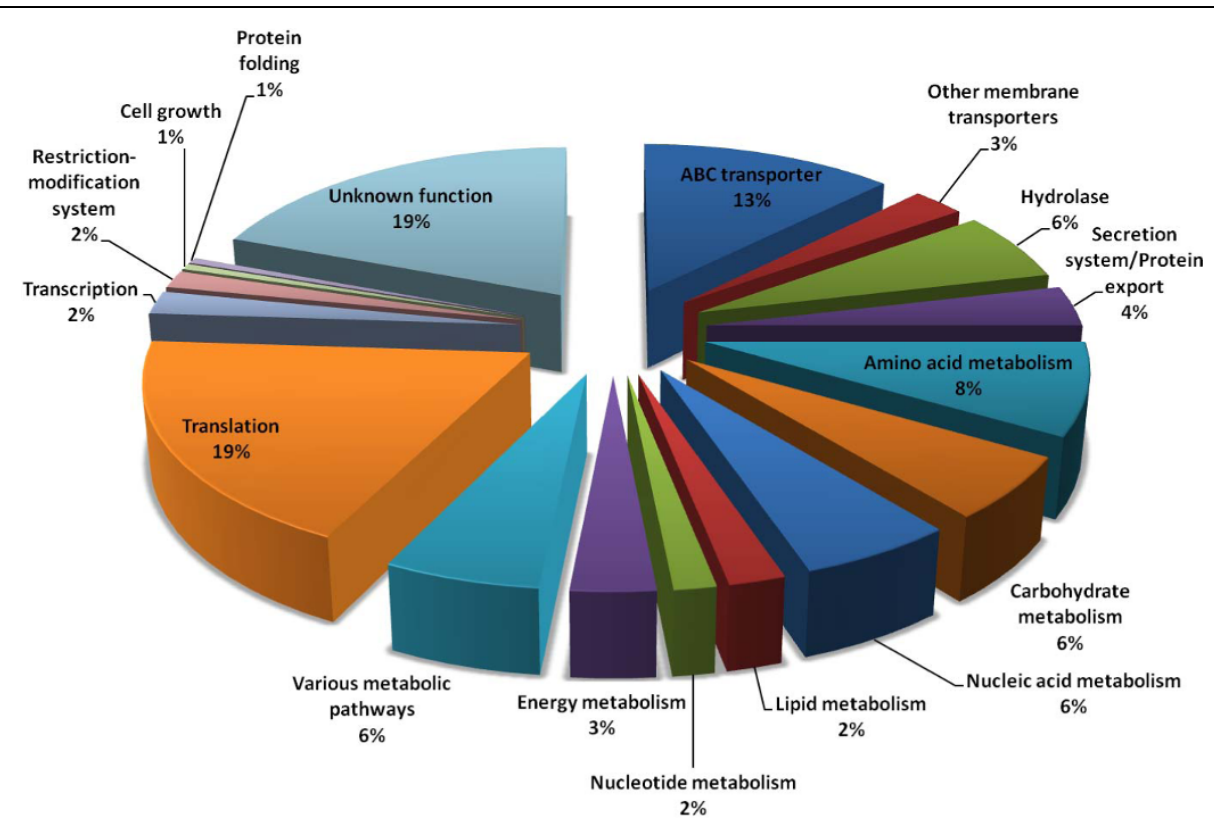

Figure 6 GO graph of proteins identified by GeLC-MS/MS in the Triton X-114 fraction of $M$. agalactiae PG2 ${ }^{\top}$. Protein identifications are classified according to function. 
for positioning/exposing lipoproteins on the outer side of the bacterial cell. About 19\% of proteins could not be assigned a specific function by manual searches or GO classification.

Proteomic data were analyzed in order to investigate presence of liposoluble proteins resulting from expression of horizontally-transferred genes [24]. Among 194 identified proteins, 15 (7.8\%) were acquired by HGT from the Mycoplasma mycoides cluster (Additional file 8), while 7 (3.7\%) were acquired by HGT from other bacteria (Additional file 9), for a total of 22 proteins, making up to $11.5 \%$ of all expressed membrane proteins being derived from putative HGT events.

\section{Discussion}

Gathering proteomic information on prokaryotic membranes is a challenging task, due to difficulties in cell fractionation and to the intrinsic chemical properties of membrane proteins in general. Therefore, both systematic and differential proteomic information on prokaryotic membranes is generally lacking. In this work, we approached the systematic characterization of what is believed to be one of the simplest bacterial pathogen membranes, in an attempt to move a step forward in our understanding of its composition, complexity, and function. In addition to its lower complexity, investigating membrane composition and plasticity in mycoplasmas is of particular interest since surface proteins are subjected to size and phase variation, and information on the extent and level of such variation is crucial in studies targeting identification of common immunogens, evaluation of immunological escape mechanisms, and adaptation of the bacterium to its host. All six variable surface lipoproteins encoded in the $\mathrm{PG2}^{\mathrm{T}}$ genome [37] were detected by 2-D PAGE, although one of these (VpmaY) was not expressed in a field isolate examined by 2D DIGE. Triplicate experiments showed that the two-dimensional expression pattern of each field isolate is relatively stable under laboratory conditions, and that there is a reproducible differential expression of several protein spots in the field isolates compared to the type strain PG2. Interestingly, these differences are being detected in bacteria which were grown in culture media, where all protein variants should theoretically be expressed [37]. It was already demonstrated that the switching mechanism is so fast that it can be pointed out in a single colony on solid culture [14]. This might suggest that the lack of VpmaY in the isolate Nurri could result from a local genetic mutation. A large-scale study performed on a higher number of field isolates might enable the detection of constantly expressed proteins, which might be useful as targets for the development of vaccines and diagnostic tools for CA.
Mycoplasmas have evolved a parasitic lifestyle, and membrane transporters are consequently very important for uptake of nutrients and growth factors. The genome of $M$. agalactiae $\mathrm{PG} 2^{\mathrm{T}}$ encodes $18 \mathrm{ABC}$ transporters, but proteins from only 10 of these were identified in this study. We also failed to identify all the components of a complete membrane transporter complex; however, it is possible that expression of all sequences encoded by the transporter gene operon may not necessarily take place at the same time. ABC transporters components encoded by different operons may likely interact to form functional transporters, producing the further advantage of creating many different combinations that can help evasion of host defense mechanisms. For instance, the genome of $M$. agalactiae $\mathrm{PG} 2^{\mathrm{T}}$ encodes for two oligopeptide (Opp) ABC transporters, one typical of the hominis group and one probably transferred by means of horizontal gene transfer mechanisms from $M$. mycoides subsp. mycoides and M. capricolum subsp. capricolum. We identified the substrate binding protein (OppA) from one operon, and the permease (OppC) and the ATP-binding protein $(\mathrm{OppF})$ from another operon; notably, these proteins create a functional transporter. Moreover, OppA could be more than a simple substrate binding protein, since it was demonstrated to play an important role in pathogenicity in M. hominis by inducing ATP release and cell death of HeLa cells in vitro and by mediating adhesion to host cells [38-40]. Other authors reported a different pattern of expression of these operons: in the study by Nouvel and co-workers [37], only OppA, OppF, and OppD were detected. These apparently controversial results could be due to technical issues, or be dependent on variations in expression of Opps within the $\mathrm{PG} 2^{\mathrm{T}}$ strain. This will need to be elucidated in future studies.

Upon analysis of all MS data, the proteins putatively assigned by the GO software as cytoplasmic accounted to $36 \%$. Among these, many hydrolases were present. However, lipases, peptidases, and nucleases might be associated to the membrane compartment and assist in reducing macromolecules to simple components, enabling their uptake. In fact, mycoplasmas lack many biosynthetic pathways and rely on internalization of nucleotides, amino acids, sugars and lipids from their external environment. Recently, it was reported that hydrolytic enzymes are surface-located in mycoplasmas, and that they can be associated with $\mathrm{ABC}$ transporters in order to digest macromolecules before uptake of simpler components, or play major roles in pathogenicity [41]. Interestingly, in the $M$. agalactiae genome, the genes coding for many of these hydrolases are also located close to $\mathrm{ABC}$ transporter operons.

Several other proteins have a predicted cytoplasmic localization, but could be membrane-associated in 
mycoplasmas, such as the elongation factor tu (EF-Tu) and the E1 beta subunit of the pyruvate dehydrogenase complex. Traditionally, these are considered to be cytoplasmic proteins involved in protein synthesis and energy production, respectively, but it was demonstrated that in M. pneumoniae they are surface exposed and interact with host fibronectin, mediating adhesion $[42,43]$. It was also demonstrated that many "cytoplasmic" proteins such as the EF-Tu are strong antigens in many mycoplasma species $[22,44,45]$.

Ribosomal proteins represent a significant proportion of the mycoplasma liposoluble proteome. This might appear inconsistent, but in spite of their traditionally cytoplasmic localization, it was already demonstrated that ribosomes interact with the bacterial protein export complex [46]. Moreover, it is well known that in eukaryotes ribosomes are associated with endoplasmic reticulum, where they participate in the protein secretion pathway [47]. Several proteins that take part in other metabolic pathways were also identified in the liposoluble fraction of $M$. agalactiae $\mathrm{PG} 2^{\mathrm{T}}$. We could speculate that many proteins involved in nutrient metabolism might associate with proteins devoted to internalization of precursors in metabolizing complexes, and be co-purified with these. Nonetheless, a pre-fractionation of membranes was not performed because of inherent technical difficulties, and we cannot rule out that enzymes with high hydrophobicity might be present as cytoplasmic contaminants.

The recent work by Sirand-Pugnet and coworkers revealed the occurrence of horizontal gene transfer (HGT) events in M. agalactiae. The expression of proteins acquired by HGT highlights the importance of horizontal gene flow for the evolutionary plasticity of mycoplasmas; for instance, by allowing changes in host and/or tissue tropism through acquisition of traits enabling colonization and survival in new niches [24,48]. In total, an impressing $11.7 \%$ of proteins expressed on the $M$. agalactiae membrane are coming from other bacteria, reinforcing the view that an important part in the evolution of mycoplasmas might be driven by genetic exchange with bacteria sharing the same host districts, probably in order to compensate the concurrent process of gene loss [24]. Another interesting observation was the detection of MAG_2340, a hypothetical lipoprotein which is apparently the result of an horizontal gene transfer event with mycoplasmas of the mycoides cluster (Additional file 8), which was not detected by Nouvel et al. in the $\mathrm{PG} 2^{\mathrm{T}}$ liposoluble proteome [37].

Hypothetical proteins were of particular interest; since these did not have an assigned function, similarity searches were conducted with BLAST tools in order to infer their possible role in the biology of mycoplasmas.
Among these, the hypothetical lipoprotein MAG_1670 belongs to the mycoides cluster LppA/P72 family, and it is an antigen recognized early and persistently in infection [49]. The hypothetical protein MAG_0250 has an indigoidine synthase A (IdgA)-like domain similar to Clostridium spp. IdgA is involved in the biosynthesis of indigoidine, a blue pigment synthesized by Erwinia chrysanthemi and implicated in pathogenicity and protection from oxidative stress by scavenging oxygen radicals [50]. Indigoidine production increases tolerance to oxidative stress and contributes to aggressiveness, and might therefore act as a virulence factor.

\section{Conclusions}

2-D PAGE studies might be extremely powerful for comparison of protein expression in different mycoplasma isolates, especially when considering that lipoproteins can be selectively detected with this method, and that size and phase variations can be easily spotted through the application of powerful differential comparison approaches as the 2D DIGE. However, these need to be integrated with traditional Western immunoblotting and GeLC-MS/MS for a deeper coverage and characterization of other mycoplasmal surface immunogens to be used as tools for vaccination, diagnosis, and therapy. This combined approach allowed the identification and characterization of $194 \mathrm{M}$. agalactiae proteins putatively localized on the membrane or associated to it, providing useful insights on its composition. In the future, alternative approaches such as blue native electrophoresis and chemical crosslinking of surface proteins will also enable to elucidate functional and structural aspects of membrane proteins that cannot be accounted for by the traditional gel-based proteomic approaches.

\section{Methods}

\section{Bacterial strains and culture conditions}

At least three replicate cultures of Mycoplasma agalactiae $\mathrm{PG}^{\mathrm{T}}$ and two Sardinian field isolates (named Bortigali and Nurri), were grown in PPLO medium supplemented with $20 \%$ heat inactivated horse serum and $500 \mu \mathrm{g} / \mathrm{mL}$ ampicillin, at $37^{\circ} \mathrm{C}$ with constant agitation. Mycoplasmas were collected by centrifugation $(10 \mathrm{~min}$ at $10,000 \times \mathrm{g}$ at $4^{\circ} \mathrm{C}$ ), and washed three times with PBS. At least three mycoplasma pellets were obtained from each bacterial culture replicate, and used for genetic and proteomic analyses. Total DNA was extracted from a set of pellets with DNeasy Blood \& Tissue Kit (Qiagen), and subjected to FS1-FS2 PCR for species confirmation [51].

\section{Total protein extracts and Triton $\mathrm{X}-114$ fractionation}

For total protein extracts, bacterial pellets were resuspended in $1 \%$ hot SDS, incubated for 3 minutes at $95^{\circ} \mathrm{C}$, chilled, and diluted with lysis buffer $(7 \mathrm{M}$ urea, $2 \mathrm{M}$ 
thiourea, 2.5\% CHAPS, 2\% ASB-14, $40 \mathrm{mM}$ Tris- $\mathrm{HCl}$ $\mathrm{pH}$ 8.8, 1\% IPG-buffer, protease inhibitors), and insoluble materials were discarded by centrifugation $(10 \mathrm{~min}$ at $10,000 \times \mathrm{g}$ at $4^{\circ} \mathrm{C}$ ) [52]. Hydrophilic and hydrophobic protein fractions were obtained by Triton X-114 fractionation $[29,30]$ and ProteoPrep Membrane Extraction Kit (Sigma-Aldrich). Proteins samples were quantified as described [52].

\section{SDS-PAGE and 2-D PAGE}

SDS-PAGE was performed on $8 \%$ polyacrylamide gels on a Protean Tetra Cell (Bio-Rad) following the manufacturer instructions, and gels were stained with PageBlue $^{\mathrm{ma}}$ Protein Staining Solution (Fermentas).

Prior to 2-D PAGE, Triton X-114 fractions were precipitated with methanol-chloroform [35] and resuspended in lysis buffer (8 M urea, $2 \mathrm{M}$ thiourea, 2.5\% CHAPS, 2\% ASB-14, $40 \mathrm{mM}$ Tris- $\mathrm{HCl} \mathrm{pH} 8.8$, 1\% IPGbuffer, protease inhibitors). Resuspended proteins (150 $\mu \mathrm{g}$ ) were then absorbed overnight into $18 \mathrm{~cm}$ IPG strips (GE Healthcare, pH 3-10 NL, pH 7-11, and pH 4-7). Strips were focused on an IPGphor (GE Healthcare) for a total of $60,000 \mathrm{Vh}$. After focusing, strips were equilibrated in $50 \mathrm{mM}$ Tris- $\mathrm{HCl}, \mathrm{pH} 6.8,2 \%$ SDS, $7 \mathrm{M}$ urea, $10 \%$ glycerol, supplemented with $2 \%$ DTT for $15 \mathrm{~min}$, and then with $2.5 \%$ iodoacetamide for $15 \mathrm{~min}$. The second dimension (SDS-PAGE) was conducted on $10 \%$ to $18 \%$ polyacrylamide gradient gels, on an Ettan DALTsix electrophoresis system (GE Healthcare), following manufacturer's instructions. 2-D gels were silver stained with a mass-compatible method [53] and images were digitalized with an Image Scanner (GE Healthcare).

\section{D DIGE}

For 2D DIGE analysis, the two field isolates Bortigali and Nurri were compared to PG2 ${ }^{\mathrm{T}}$. Triton X-114 Protein extracts were precipitated and resuspended in lysis buffer as described above. Then, samples were labeled with CyDye DIGE Fluors (GE Healthcare) according to the minimal labeling protocol provided by the manufacturer. Briefly, after CyDye reconstitution with dimethylformamide (DMF) and preparation of a working solution $(200 \mathrm{pmol} / \mu \mathrm{L}), 1 \mu \mathrm{L}$ of diluted CyDye was added to a volume of protein sample equivalent to 50 $\mu \mathrm{g}$. Samples were left on ice for 30 minutes in the dark, and then $1 \mu \mathrm{L}$ of $10 \mathrm{mM}$ lysine was added to stop the reaction. Labeled samples were mixed, IPG buffer corresponding to the desired $\mathrm{pH}$ range was added at a $1 \%$ final concentration, and DeStreak Rehydration Solution (GE Healthcare) was added to a total volume of $340 \mu \mathrm{L}$. $18 \mathrm{~cm}$ IPG strips (GE Healthcare) were passively rehydrated for at least 6 hours. IEF was carried out on an Ettan IPGphor II (GE Healthcare) for a total of $~ 60,000$ Vh. After focusing, strips were equilibrated in $50 \mathrm{mM}$
Tris- $\mathrm{HCl}, \mathrm{pH}$ 6.8, 2\% SDS, $7 \mathrm{M}$ urea, 10\% glycerol, supplemented with $2 \%$ DTT for $10 \mathrm{~min}$, and then with $2.5 \%$ iodoacetamide for $10 \mathrm{~min}$. Proteins were then subjected to SDS-PAGE in 10-18\% gradient polyacrylamide gels on the Ettan DALTsix system (GE Healthcare), following the manufacturer instructions. DIGE images were detected with a Typhoon Scanner (GE Healthcare) and processed with DeCyder (GE Healthcare) for image analysis.

\section{Western Immunoblotting}

SDS-PAGE resolved proteins were transferred onto nitrocellulose membranes with a Mini-Trans-Blot Cell (Bio-Rad) at $250 \mathrm{~mA}$ for one hour. After blotting, membranes were blocked with PBS-0.05\% Tween 20 (PBS-T) containing 3\% BSA. Membranes were incubated for one hour with a rabbit hyperimmune serum raised against $M$. agalactiae recombinant $\mathrm{P} 48$ (M. agalactiae $\mathrm{rP} 48$ ) [12]. Membranes were washed five times with PBS-T and incubated with the appropriate HRP-conjugated secondary antibodies (Sigma). After five washes, membranes were developed with SuperSignal West Pico Chemiluminescent Substrate (Pierce) and images were acquired with a VersaDoc MP 4000 Imaging System (Bio-Rad).

\section{Spot picking and in situ tryptic digestion}

Protein spots obtained upon 2-D PAGE separation of the Triton $\mathrm{X}-114$ extract from the strain $\mathrm{PG}^{\mathrm{T}}$ were manually excised from gels, destained with $15 \mathrm{mM} \mathrm{K}_{3} \mathrm{Fe}$ $(\mathrm{CN})_{6}$ in $50 \mathrm{mM} \mathrm{Na} \mathrm{S}_{2} \mathrm{O}_{3}$ and stored in acetonitrile. Spots were then subjected to an $\mathrm{O} / \mathrm{N}$ tryptic digestion at $37^{\circ} \mathrm{C}$ in $50 \mathrm{mM}\left(\mathrm{NH}_{4}\right) \mathrm{HCO}_{3}, \mathrm{pH} 8.0$, using 40 to $80 \mathrm{ng}$ of trypsin depending on spot intensity. Peptide mixtures were collected by elution with acetonitrile followed by centrifugation. Peptides were then acidified with TFA $20 \%$, dried in SpeedVac ${ }^{\circ}$, resuspended in $0.2 \%$ formic acid and stored at $-20^{\circ} \mathrm{C}$.

\section{GeLC-MS/MS}

The Triton X-114 fraction was diluted with $4 \times$ Laemmli buffer [54], $20 \mu \mathrm{g}$ of proteins were loaded in an $8 \%$ polyacrylamide gel, and SDS-PAGE was performed as previously described. After gel staining, bands were manually excised, destained, reduced, alkylated, and finally subjected to in situ tryptic digestion as previously described [55]. Peptide mixtures were identified by nanoHPLC-nanoESI-Q-TOF-analysis. One-dimensional patterns were analyzed with Quantity One software (Bio-Rad).

\section{MALDI-MS}

Mass spectra were recorded on a MALDI micro (Waters, Manchester, UK) equipped with a reflectron analyzer and used in delayed extraction mode, as 
described previously [56]. Peptide samples were mixed with an equal volume of $\alpha$-cyano-4-hydroxycynnamic acid as matrix $(10 \mathrm{mg} / \mathrm{mL}$ in acetonitrile/0.2\% TFA) (70:30, v/v), applied to the metallic sample plate, and air dried. Mass calibration was performed by using the standard mixture provided by manufacturer. Raw data, reported as monoisotopic masses, were then introduced into the in-house Mascot Peptide Mass Fingerprinting software (Version 2.2, Matrix Science, Boston, MA), and used for protein identification. Search parameters were as follows: fixed modifications carbamidomethyl (C), variable modifications pyro-Glu $(\mathrm{N}$-term $\mathrm{Q})$ and oxidation $(\mathrm{M})$, peptide tolerance 80 ppm, enzyme trypsin, allowing up to 2 missed cleavages.

\section{LC-MS/MS}

LC-MS/MS analyses of tryptic digests were performed on a Q-TOF hybrid mass spectrometer equipped with a nano lock Z-spray source, and coupled on-line with a capillary chromatography system CapLC (Waters, Manchester, UK), as described previously [55]. After loading, the peptide mixture was first concentrated and washed at $20 \mu \mathrm{L} /$ min onto a reverse-phase pre-column (Symmetry 300, C18, $5 \mu \mathrm{m}$, NanoEase, Waters) using $0.2 \%$ formic acid as eluent. The sample was then fractionated onto a C18 reverse-phase capillary column (Nanoflow column $5 \mu \mathrm{m}$ Biosphere C18, $75 \mu \mathrm{m} \times 200 \mathrm{~mm}$, Nanoseparations) at a flow rate of $250 \mathrm{~nL} / \mathrm{min}$, using a linear gradient of eluent B ( $0.2 \%$ formic acid in $95 \%$ acetonitrile) in A ( $0.2 \%$ formic acid in 5\% acetonitrile) from 2 to $40 \%$ in $27 \mathrm{~min}$. The mass spectrometer was set up in a data-dependent MS/ MS mode where a full scan spectrum $(\mathrm{m} / \mathrm{z}$ acquisition range from 400 to $1600 \mathrm{Da} / \mathrm{e}$ ) was followed by tandem mass spectra (m/z acquisition range from 100 to $2000 \mathrm{Da} /$ e). Peptide ions were selected as the three most intense peaks of the previous scan. A suitable collision energy was applied depending on the mass and charge of the precursor ion. Argon was used as the collision gas. Mass calibration was conducted on the Glu-fibrino peptide B (Sigma) fragmentation pattern. ProteinLynx software (Version 2.2.5), provided by the manufacturers, was used to analyze raw MS and MS/MS spectra and to generate a peak list which was introduced in the in-house Mascot MS/MS ion search software (Version 2.2, Matrix Science, Boston, MA) for protein identification. NCBI was used as sequence database. Search parameters were as follows: fixed modifications carbamidomethyl (C), variable modifications pyroGlu (N-term Q) and oxidation (M), peptide tolerance 30 ppm, MS/MS tolerance $0.3 \mathrm{Da}$, charge state +2 and +3 , enzyme trypsin, allowing up to 1 missed cleavage.

\section{Data analysis}

MS data were subjected to gene ontology analysis with Blast2GO, using default parameters [57]. Identified proteins were divided into classes for functional and localization analysis; data produced by the software were used for generation of graphs by Microsoft Excel.

\section{Additional material}

\section{Additional file 1: 2-D PAGE map of liposoluble proteins from $M$. agalactiae $P G 2^{\top}$ illustrating the protein identifications obtained by MS on the 3-10NL $p$ I Interval. \\ Additional file 2: 2-D PAGE map of liposoluble proteins from $M$. agalactiae $\mathrm{PG}^{\top}{ }^{\top}$ illustrating the protein identifications obtained by MS on the 7-11 pl Interval \\ Additional file 3: 2-D PAGE map of liposoluble proteins from $M$. agalactiae $\mathrm{PG}^{\top}{ }^{\top}$ illustrating the protein identifications obtained by MS on the 4-7 pl Interval.}

Additional file 4: Table listing all protein identifications obtained from 2-D PAGE maps. The proteins listed in this table were identified from 2-D PAGE maps of the $M$. agalactiae $P G 2^{\top}$ Triton $X-114$ fraction. Maps are represented in Additional files 1 ( $\mathrm{pH} 3-10 \mathrm{NL}), 2$ (pH 7-11) and 3 $(\mathrm{pH} 4-7)$

Additional file 5: Protein profile of liposoluble proteins before and after precipitation. Right: approach used for GeLC-MS/MS

characterization. The bars indicate the regions cut from the PAGE gel and subjected to mass spectrometry characterization. Protein identifications are reported in additional file 6, from top to bottom.

Additional file 6: Table listing all protein identifications obtained by GeLC-MS/MS of the M. agalactiae PG2 ${ }^{\top}$ Triton X-114 liposoluble fraction. The protein profile used and the number of slices are reported in Additional file 5 .

Additional file 7: Functional analysis, number of peptide hits, and method of detection of $M$. agalactiae $\mathrm{PG}^{\top}{ }^{\top}$ liposoluble proteins. The results of $2 \mathrm{D}$ DIGE with the two field strains Nurri and Bortigali are also reported (TPH, total peptide hits; NA, not applicable).

Additional file 8: Proteins identified in the $M$. agalactiae proteome potentially resulting from Horizontal Gene Transfer events with $M$. mycoides subsp. mycoides and $M$. capricolum subsp. capricolum.

Additional file 9: Proteins identified in the $M$. agalactiae proteome potentially resulting from Horizontal Gene Transfer events with other bacteria.

\section{Acknowledgements}

We thank Prof. Christine Citti for kindly providing the type strain $P G 2^{\top}$, Dr. Mario Ferrer-Navarro for his helpful suggestions during optimization of the protein fractionation approach, Dr. Vittorio Tedde and Dr. Alessandro Tanca for assistance during electrophoresis and MALDI-MS identification, and Dr. Stefania Ghisaura from Biosistema Scarl for the DIGE experiments. This work was supported by funding from the Grant "Ricerca Sanitaria Finalizzata, Anno 2007, UPB S02.04.010, Cap SC02.1106", and Misura P5 Biodiversità animale (Regione Sardegna).

\section{Author details}

'Dipartimento di Patologia e Clinica Veterinaria, Università degli Studi di Sassari, Sassari, Italy. ${ }^{2}$ Porto Conte Ricerche Srl, Tramariglio, Alghero (SS), Italy. ${ }^{3}$ Dipartimento di Scienze Biomediche, Università degli Studi di Sassari, Sassari, Italy.

\section{Authors' contributions}

CC and MFA performed the experimental design, carried out the protein fractionation and electrophoresis, performed data analysis, and drafted the manuscript. DP carried out the mass spectrometry identifications. BC participated in the design of the study. EC and LC performed animal diagnosis, collection of animal samples, isolation, molecular identification, and cultivation of mycoplasmas. SU contributed to coordination of the study and data interpretation, and helped to draft the manuscript. AA and MP 
conceived the study, participated in its design and coordination, and helped to draft the manuscript. All authors read and approved the final manuscript.

Received: 4 May 2010 Accepted: 25 August 2010

Published: 25 August 2010

\section{References}

1. Razin S, Yogev D, Naot Y: Molecular biology and pathogenicity of mycoplasmas. Mol Biol Rev 1998, 62:1094-1156.

2. Rottem S: Interaction of mycoplasmas with host cells. Physiol Rev 2003, 83:417-432.

3. You XX, Zeng YH, Wu YM: Interactions between mycoplasma lipidassociated membrane proteins and the host cells. J Zhejiang Univ Sci B 2006, 7:342-350.

4. Kühner S, van Noort V, Betts MJ, Leo-Macias A, Batisse C, Rode M, Yamada T, Maier T, Bader S, Beltran-Alvarez P, Castaño-Diez D, Chen WH, Devos D, Güell M, Norambuena T, Racke I, Rybin V, Schmidt A, Yus E, Aebersold R, Herrmann R, Böttcher B, Frangakis AS, Russell RB, Serrano L, Bork P, Gavin AC: Proteome organization in a genome-reduced bacterium. Science 2009, 27:1235-1240.

5. Lambert M: Contagious agalactia of sheep and goats. Rev Sci Tech OIE 1987, 6:699-711, Mycoplasmoses of ruminants.

6. Corrales JC, Esnal A, De la Fe C, Sánchez A, Assunçao P, Poveda JB, Contreras A: Contagious agalactia in small ruminants. Small Rum Res 2007, 68:154-166.

7. Bergonier D, Berthelot X, Poumarat F: Contagious agalactia of small ruminants: current knowledge concerning epidemiology, diagnosis and control. Rev Sci Tech Off Int Epizoot 1997, 16:848-873.

8. Chessa B, Pittau M, Puricelli M, Zobba R, Coradduzza E, Dall'ara P, Rosati S, Poli G, Alberti A: Genetic immunization with the immunodominant antigen P48 of Mycoplasma agalactiae stimulates a mixed adaptive immune response in BALBc mice. Res Vet Sci 2009, 86:414-420.

9. Fusco M, Corona L, Onni T, Marras E, Longheu C, Idini G, Tola S Development of a sensitive and specific enzyme-linked immunoadsorbent assay based on recombinant antigens for rapid detection of antibodies against Mycoplasma agalactiae in sheep. Clin Vaccine Immunol 2007, 14:420-425.

10. Greco G, Corrente M, Buonavoglia D, Aliberti A, Fasanella A: Inactivated vaccine induces protection against Mycoplasma agalactiae infection in sheep. New Microbiol 2002, 25:17-20.

11. Nicholas RAJ: Contagious agalactia and other mycoplasmal mastitides of small ruminants. In The Merck Veterinary Manual. Edited by: Kahn CM, Line S. Merck , 9 2005:1114-1116.

12. Rosati S, Robino P, Fadda M, Pozzi S, Mannelli A, Pittau M: Expression and antigenic characterization of recombinant Mycoplasma agalactiae P48 major surface protein. Vet Microbiol 2000, 71:201-210.

13. Tola S, Manunta D, Rocca S, Rocchigiani AM, Idini G, Angioi PP, Leori G: Experimental vaccination against Mycoplasma agalactiae using different inactivated vaccines. Vaccine 1999, 10:2764-2768.

14. Chopra-Dewasthaly R, Citti C, Glew MD, Zimmermann M, Rosengarten R, Jechlinger W: Phase-locked mutants of Mycoplasma agalactiae: defining the molecular switch of high-frequency Vpma antigenic variation. Mol Microbiol 2008, 67:1196-1210.

15. McAuliffe L, Kokotovich B, Ayling RD, Nicholas RA: Molecular epidemiological analysis of Mycoplasma bovis isolates from the United Kingdom shows two genetically distinct clusters. J Clin Microbiol 2004, 42:4556-4565.

16. Citti C, Watson-McKown R, Droesse M, Wise KS: Gene families encoding phase- and size-variable surface lipoproteins of Mycoplasma hyorhinis. $J$ Bacteriol 2000, 182:1356-1363.

17. Glew MD, Papazisi L, Poumarat F, Bergonier D, Rosengarten R, Citti C: Characterization of a multigene family undergoing high-frequency DNA rearrangements and coding for abundant variable surface proteins in Mycoplasma agalactiae. Infect Immun 2000, 68:4539-4548.

18. Fleury B, Bergonier D, Berthelot X, Peterhans E, Frey J, Vilei EM: Characterization of P40, a cytadhesin of Mycoplasma agalactiae. Infect Immun 2002, 70:5612-5621.

19. Fleury B, Bergonier D, Berthelot $X$, Schlatter $Y$, Frey J, Vilei EM: Characterization and analysis of a stable serotype-associated membrane protein (P30) of Mycoplasma agalactiae. J Clin Microbiol 2001, 39:2814-2822.
20. Rosati S, Pozzi S, Robino P, Montinaro B, Conti A, Fadda M, Pittau M: P48 major surface antigen of Mycoplasma agalactiae is homologous to a malp product of Mycoplasma fermentans and belongs to a selected family of bacterial lipoproteins. Infect Immun 1999, 67:6213-6216.

21. Tola S, Crobeddu S, Chessa G, Uzzau S, Idini G, Ibba B, Rocca S: Sequence, cloning, expression and characterisation of the $81-\mathrm{kDa}$ surface membrane protein (P80) of Mycoplasma agalactiae. FEMS Microbiol Lett 2001, 202:45-50.

22. Jores J, Meens J, Buettner FF, Linz B, Naessens J, Gerlach GF: Analysis of the immunoproteome of Mycoplasma mycoides subsp. mycoides small colony type reveals immunogenic homologues to other known virulence traits in related Mycoplasma species. Vet Immunol Immunopathol 2009, 131:238-245.

23. Minion FC: Mycoplasma gene expression in Escherichia coli. Methods Mol Biol 1998, 104:259-265.

24. Sirand-Pugnet $P$, Lartigue $C$, Marenda $M$, Jacob $D$, Barré $A$, Barbe $V$, Schenowitz C, Mangenot S, Couloux A, Segurens B, de Daruvar A, Blanchard A, Citti C: Being pathogenic, plastic, and sexual while living with a nearly minimal bacterial genome. PLoS Genet 2007, 3:e75.

25. Görg A, Weiss W, Dunn MJ: Current two-dimensional electrophoresis technology for proteomics. Proteomics 2004, 4:3665-3685.

26. Razin S: Peculiar properties of mycoplasmas: The smallest self-replicating prokaryotes. FEMS Microbiol Lett 1992, 15:423-431.

27. Regula JT, Ueberle B, Boguth G, Görg A, Schnölzer M, Herrmann R, Frank R: Towards a two-dimensional proteome map of Mycoplasma pneumoniae. Electrophoresis 2000, 21:3765-3780.

28. Wasinger VC, Pollack JD, Humphery-Smith I: The proteome of Mycoplasma genitalium. Chaps-soluble component. Eur J Biochem 2000, 267:1571-1582.

29. Bordier C: Phase-separation of integral membrane proteins in Triton X114 solution. J Biol Chem 1981, 25:1604-1607.

30. Pittau M, Fadda M, Briguglio P: Triton X-114 phase fractionation of Mycoplasma agalactiae membrane proteins and affinity purification of specific antibodies. Atti Soc Ital Sci Vet 1990, 44:925-928.

31. Donoghue PM, Hughes C, Vissers JP, Langridge Jl, Dunn MJ: Nonionic detergent phase extraction for the proteomic analysis of heart membrane proteins using label-free LC-MS. Proteomics 2008, 8:3895-3905.

32. Li YZ, Ho YP, Chen ST, Chiou TW, Li ZS, Shiuan D: Proteomic comparative analysis of pathogenic strain 232 and avirulent strain J of Mycoplasma hyopneumoniae. Biochemistry (Mosc) 2009, 74:215-220.

33. Marouga R, David S, Hawkins E: The development of the DIGE system: $2 D$ fluorescence difference gel analysis technology. Anal Bioanal Chem 2005, 382:669-678.

34. Timms JF, Cramer R: Difference gel electrophoresis. Proteomics 2008, 8:4886-4897.

35. Ünlü M, Morgan ME, Minden JS: Difference gel electrophoresis: A single method for detecting changes in protein extracts. Electrophoresis 1997, 18:2071-2077.

36. Schirle M, Heurtier MA, Kuster B: Profiling core proteomes of human cell lines by one-dimensional PAGE and liquid chromatography-tandem mass spectrometry. Mol Cell Proteomics 2003, 2:1297-1305

37. Nouvel $L X$, Sirand-Pugnet $P$, Marenda MS, Sagné E, Barbe $V$, Mangenot $S$, Schenowitz C, Jacob D, Barré A, Claverol S, Blanchard A, Citti C: Comparative genomic and proteomic analyses of two Mycoplasma agalactiae strains: clues to the macro- and micro-events that are shaping mycoplasma diversity. BMC Genomics 2010, 2:11-86.

38. Henrich B, Hopfe M, Kitzerow A, Hadding U: The adherence-associated lipoprotein P100, encoded by an opp operon structure, functions as the oligopeptide-binding domain OppA of a putative oligopeptide transport system in Mycoplasma hominis. J Bacteriol 1999, 181:4873-4878.

39. Hopfe M, Henrich B: OppA, the substrate-binding subunit of the oligopeptide permease, is the major Ecto-ATPase of Mycoplasma hominis. J Bacteriol 2004, 186:1021-1028.

40. Hopfe M, Henrich B: OppA, the ecto-ATPase of Mycoplasma hominis induces ATP release and cell death in HeLa cells. BMC Microbiol 2008, 8:55.

41. Schmidt JA, Browning GF, Markham PF: Mycoplasma hyopneumoniae mhp379 is a $\mathrm{Ca}^{2+}$-dependent, sugar-nonspecific exonuclease exposed on the cell surface. J Bacterio/ 2007, 189:3414-3424.

42. Balasubramanian S, Kannan TR, Baseman JB: The surface-exposed carboxyl region of Mycoplasma pneumoniae elongation factor Tu interacts with fibronectin. Infect Immun 2008, 76:3116-3323. 
43. Dallo SF, Kannan TR, Blaylock MW, Baseman JB: Elongation factor Tu and E1 beta subunit of pyruvate dehydrogenase complex act as fibronectin binding proteins in Mycoplasma pneumoniae. Mol Microbiol 2002, 46:1041-1051.

44. Alonso JM, Prieto M, Parra F: Genetic and antigenic characterisation of elongation factor Tu from Mycoplasma mycoides subsp. mycoides SC. Vet Microbiol 2002, 89:277-289.

45. Bercic RL, Slavec B, Lavric M, Narat M, Bidovec A, Dovc P, Bencina D: Identification of major immunogenic proteins of Mycoplasma synoviae isolates. Vet Microbiol 2008, 127:147-54.

46. Johnson AE: The structural and functional coupling of two molecular machines, the ribosome and the translocon. J Cell Biol 2009, 185:765-767.

47. White $\mathrm{SH}$, von Heijne G: How translocons select transmembrane helices. Annu Rev Biophys 2008, 37:23-42.

48. Marenda M, Barbe V, Gourgues G, Mangenot S, Sagne E, Citti C: A new integrative conjugative element occurs in Mycoplasma agalactiae as chromosomal and free circular forms. J Bacteriol 2006, 188:4137-4141.

49. Cheng $X$, Nicolet J, Miserez R, Kuhnert $P$, Krampe M, Pilloud T, Abdo EM, Griot C, Frey J: Characterization of the gene for an immunodominant 72 kDa lipoprotein of Mycoplasma mycoides subsp. mycoides small colony type. Microbiology 1996, 142:3515-3524.

50. Reverchon S, Rouanet C, Expert D, Nasser W: Characterization of Indigoidine Biosynthetic Genes in Erwinia chrysanthemi and Role of This Blue Pigment in Pathogenicity. J Bacteriol 2002, 184:654-665.

51. Tola S, Idini G, Manunta D, Galleri G, Angioi A, Rocchigiani AM, Leori G: Rapid and specific detection of Mycoplasma agalactiae by polymerase chain reaction. Vet Microbiol 1996, 51:77-84.

52. Ferrer-Navarro M, Gómez A, Yanes $\mathrm{O}$, Planell R, Avilés FX, Piñol J, Pérez J, Pons A, Querol E: Proteome of the bacterium Mycoplasma penetrans. J Proteome Res 2006, 5:688-694.

53. Chevallet M, Luche S, Rabilloud T: Silver staining of proteins in polyacrylamide gels. Nat Protoc 2006, 1:1852-1858.

54. Laemmli UK: Cleavage of structural proteins during the assembly of the head of bacteriophage T4. Nature 1970, 227:680-685.

55. Addis MF, Tanca A, Pagnozzi D, Crobu S, Fanciulli G, Cossu-Rocca P, Uzzau S: Generation of high-quality protein extracts from formalin-fixed, paraffin-embedded tissues. Proteomics 2009, 9:3815-3823.

56. Addis MF, Tanca A, Pagnozzi D, Rocca S, Uzzau S: 2-D PAGE and MS analysis of proteins from formalin-fixed, paraffin-embedded tissues. Proteomics 2009, 9:4329-4339.

57. Götz S, García-Gómez JM, Terol J, Williams TD, Nueda MJ, Robles M, Talón M, Dopazo J, Conesa A: High-throughput functional annotation and data mining with the Blast2GO suite. Nucleic Acids Res 2008, 36:3420-3435.

doi:10.1186/1471-2180-10-225

Cite this article as: Cacciotto et al: The liposoluble proteome of Mycoplasma agalactiae: an insight into the minimal protein complement of a bacterial membrane. BMC Microbiology 2010 10:225

\section{Submit your next manuscript to BioMed Central and take full advantage of:}

- Convenient online submission

- Thorough peer review

- No space constraints or color figure charges

- Immediate publication on acceptance

- Inclusion in PubMed, CAS, Scopus and Google Scholar

- Research which is freely available for redistribution

Submit your manuscript at www.biomedcentral.com/submit
Biomed Central 